\title{
Evolutionary dynamics of the Warburg effect: Glycolysis as a collective action problem among cancer cells
}

\author{
Marco Archetti* \\ School of Biological Sciences, University of East Anglia, Norwich Research Park, Norwich NR4 7TJ, UK
}

\section{H I G H L I G H T S}

- The upregulation of glycolysis in tumors is common even when oxygen is not limiting.

- The adaptive value of this "Warburg effect" is unclear.

- Glycolysis is costly for a cell but the ensuing acidity is beneficial for the tumor.

- A collective action problem among cancer cells arises.

- Game theory shows that the acidity induced by glycolysis can explain the Warburg effect.

\section{A R T I C L E I N F O}

\section{Article history:}

Received 1 April 2013

Received in revised form

7 September 2013

Accepted 13 September 2013

Available online 27 September 2013

\section{Keywords:}

Tumor

Game theory

Public good

Cooperation

Evolution

\begin{abstract}
A B S T R A C T
The upregulation of glycolysis in cancer cells (the "Warburg effect") is common and has implications for prognosis and treatment. As it is energetically inefficient under adequate oxygen supply, its adaptive value for a tumor remains unclear. It has been suggested that the acidity produced by glycolysis is beneficial for cancer cells because it promotes proliferation against normal cells. Current models of this acid-mediated tumor invasion hypothesis, however, do not account for increased glycolysis under nonlimiting oxygen concentrations and therefore do not fully explain the Warburg effect. Here I show that the Warburg effect can be explained as a form of cooperation among cancer cells, in which the products of glycolysis act as a public good, even when oxygen supply is high enough to make glycolysis energetically inefficient. A multiplayer game with non-linear, non-monotonic payoff functions that models the benefits of the acidity induced by glycolysis reveals that clonal selection can stabilize glycolysis even when energetically costly, that is, under non-limiting oxygen concentration. Characterizing the evolutionary dynamics of glycolysis reveals cases in which anti-cancer therapies that rely on the modification of acidity can be effective.
\end{abstract}

(c) 2013 Elsevier Ltd. All rights reserved.

\section{Introduction}

\subsection{The Warburg effect}

The upregulation of glycolysis (the conversion of glucose to pyruvate, with consequent production of ATP, which does not require oxygen) by cancer cells, first reported by Warburg (1930), is a common feature of tumors and may seem an adaptation to hypoxia, which often occurs as a consequence of the fact that oxygen concentration decreases with distance from a capillary (Krogh, 1919, Thomlinson \& Gray 1995, Dewhirst et al., 1994; Helmlinger et al., 1997). Tumors, however, consistently rely on glycolysis even in the presence of abundant oxygen (Beckner et al., 2005; Griguer et al., 2005;

\footnotetext{
* Tel.: +44 1603591241.

E-mail addresses: m.archetti@uea.ac.uk, archetti.marco@gmail.com
}

Kelloff et al., 2005; Rajendran et al., 2003). Since the anaerobic metabolism of glucose to lactic acid is substantially less efficient than oxidation to $\mathrm{CO}_{2}$ and $\mathrm{H}_{2} \mathrm{O}$, tumor cells must increase glucose flux in order to maintain sufficient ATP production. This is the basis of the detection of glycolysis with Fluoro-deoxy-D-Glucose Positron Emission Tomography (FdG PET) (Pauwels et al., 2000; Gambhir, 2002). It is now so clear that the Warburg effect occurs even under non-limiting oxygen conditions, that FdG PET is commonly used for diagnosis and for monitoring treatment.

Why do cancer cells upregulate glycolysis? Although some organisms use glycolysis preferentially during periods of sustained growth (because the byproducts are useful as building blocks in the anabolic process), glycolysis is highly inefficient when oxygen is not a limiting factor because the anaerobic metabolism of glucose to lactic acid produces fewer ATP molecules per molecule of glucose than oxidation to $\mathrm{CO}_{2}$ and $\mathrm{H}_{2} \mathrm{O}$, and therefore leads to slower proliferation. Since cancer progression is a process of clonal 
selection (Cairns, 1975; Nowell, 1976; Crespi and Summers, 2005; Merlo et al., 2006; Greaves and Maley, 2012), upregulation of glycolysis must confer a selective advantage to a cell to compensate for its slower proliferation. If it is not simply an adaptation to hypoxia, what is the adaptive value of the Warburg effect for cancer cells?

Besides its relevance to our basic understanding of cancer biology and the diagnostic and monitoring applications mentioned above, the Warburg effect has implications for treatment, as hypoxic tumors are often more invasive and metastatic than those with normal oxygen levels (Kunkel et al., 2003; Mochiki et al., 2004, Postovit et al., 2002, 2004; He et al., 2004; Buchler et al., 2003; but see also Krtolica and Ludlow, 1996), and treatments like anti-angiogenic drugs that aim at impairing the provision of oxygen to the tumor may fail if tumors can switch to glycolysis (Bergers and Hanahan, 2008).

\subsection{The acid-mediated tumor invasion hypothesis}

In a series of papers, Gatenby and others (e.g.: Gatenby and Gawlinski, 1996, 2003; Gatenby and Gillies, 2004, 2007) have suggested that the Warburg effect is a way for the tumor to increase its proliferation rate against normal cells, due to the fact that glycolysis induces microenvironmental acidification (Schornack and Gillies, 2003; Griffiths et al., 2001; Bhujwalla et al., 2002). An acidic microenvironment is known to confer an advantage to tumor cells by promoting the death of normal cells (Rubin, 1971; Dairkee et al., 1995; Casciari et al., 1992), since normal cells lack a mechanism to adapt to extracellular acidosis (such as mutations in p53 or other components of the apoptotic pathway, over-expression of NHE and autophagy (Park et al., 1999; Williams et al., 1999; Wojtkowiak et al., 2012)). Furthermore, acidity increases extra-cellular matrix degradation by proteolytic enzymes such as cathepsin B (Rohzin et al., 1994), which facilitates tumor invasiveness, it stimulates the release of vascular endothelial growth factor and interleukin 8 (Shi et al., 2001), which promote neo-angiogenesis, and inhibits immune function (Lardner, 2001).

In short, the hypothesis is that, even though glycolysis leads to slower proliferation for an individual cell, the consequent benefits for the tumor as a whole due to the acidification of the extracellular space (which results in toxicity for normal cells, promotes neo-angiogenesis and inhibits immune reaction) confers an overall proliferative advantage to the tumor. The hypothesis, if correct, has significant implications for cancer therapy, as manipulation of acidity could lead to anti-cancer effects.

Models of this acid-mediated tumor invasion hypothesis (Gatenby and Gawlinski, 1996; Patel et al., 2001; Smallbone et al. 2005, 2007; Basanta et al., 2008, 2011; Silva et al., 2010) show that cells with increased glycolysis will also evolve resistance to acidinduced toxicity, which can lead indeed to a significant proliferative advantage for the tumor. These models, however, make a crucial assumption: that resistance can only arise in cells with glycolysis (e.g.: Basanta et al., 2008) or that, even if resistance is not limited to hyperglycolytic cells, mutations are irreversible (e.g.: Smallbone et al., 2007; Silva et al., 2010), not allowing therefore resistant non-glycolytic cells to arise from resistant glycolytic cells. The problem that these models leave unsolved is that, by not allowing resistance to evolve in cells with aerobic metabolism or by assuming that resistant hyperglycolytic cells cannot mutate back to aerobic metabolism, they leave unexplained the very problem that the acid-mediated tumor invasion hypothesis wanted to address in the first place: why is higher glycolysis also observed under normal oxygen concentrations? In other words: what prevents a cell that forego glycolysis to invade a population?
If resistance to acidity arises in cancer cells with aerobic metabolism as well (and there is no compelling reason to assume otherwise), or if resistant cells can mutate back and abandon glycolysis (which is also reasonable), these cells would have more efficient metabolism than cancer cells with glycolysis, and could still exploit the benefit of acidity (against normal cells) induced by other cancer cells with glycolysis. Clonal selection occurs not just between cancer cells versus normal cells, but also between cancer cells with aerobic metabolism versus cancer cells with anaerobic metabolism. What maintains glycolysis (given its private cost) among cancer cells, if the hypothesized (public) benefit (acidity) accrues to all cancer cells, including those that forego glycolysis to revert to aerobic metabolism?

\subsection{Glycolysis as a public goods game}

The problem can be understood more easily in game-theoretic terms. Glycolysis is a private benefit under low oxygen concentrations (because it allows a cell to survive), whereas under nonlimiting oxygen concentrations it is a private cost (due to the consequent inefficient metabolism); glycolysis also produces a public good (for the cancer cells): the associated acidity (against the normal cells). The benefit of acidity accrues to all tumor cells, irrespective of whether they have aerobic or anaerobic metabolism. Cells that do not pay the cost of an inefficient metabolism could free-ride on the acidity induced by neighboring tumor cells, thereby exploiting its benefit without paying the cost. In short, glycolysis can be considered a cooperative phenotype, and reverting to aerobic metabolism can be considered free-riding. This raises a classical collective action problem: why, under normal oxygen conditions, do not cells resistant to acidity forego glycolysis and revert to the more efficient aerobic metabolism, free-riding on the benefits of acidosis produced by the other cells' glycolysis? Current models of the acid-mediated tumor invasion hypothesis do not address this problem because they assume that resistance can only arise in cells with increased glycolysis or that resistant hyperglycolytic cells cannot forego glycolysis. Allowing resistant cells to revert to aerobic metabolism would make make glycolysis inefficient, and the hypothesis would fail.

The problem can be analyzed using evolutionary game theory. There are, a number of differences between the scenario of the Warburg effect and previous game theory models, which make such analysis non trivial. First, as already observed, previous game theory models of cancer progression related to the problem of glycolysis (Basanta et al., 2008, 2011) only allow the evolution of resistance in the glycolytic type. Moreover, these models assume that interactions occur between pairs of cells. In the case of glycolysis-induced acidosis, however, as acidification depends on the diffusion of the metabolic products of glycolysis, such as lactic acid and hydrogen ions $\left(\mathrm{H}^{+}\right.$), in the extracellular space (Schornack and Gillies, 2003; Griffiths et al., 2001; Bhujwalla et al., 2002), a cell's fitness depends on the collective interactions with neighboring cells, rather than on the payoff of multiple pairwise encounters with individual cells. In other words, because the products of glycolysis act as diffusible public goods, glycolysis should be modeled as a public goods game, rather than as a game with pairwise interactions; games with pairwise interactions, even when multiple interactions are allowed, do not generally have the same results as multi-player, collective action (public goods) games. An important difference is that, while in two-player nonlinear games the maximum benefit for the population is achieved when all players cooperate, in multi-player games the best outcome for the population is achieved at intermediate frequencies of cooperators (Archetti and Scheuring, 2012). This, as we will see, has important implications for the dynamics of potential therapies based on the modification of acidosis. 
Second, even if we resort to public goods games to model the Warburg effect, there are differences between the benefit function of public goods games generally assumed in evolutionary game theory and the benefit function we must assume for cancer cells with glycolysis, which makes the analysis more complex. While evolutionary models have generally assumed a linear or a step benefit function (Archetti, 2009a,b), or synergistic and saturating benefits (Motro, 1991; Hauert et al., 2006), games with more complex non-linear benefits, such as the benefits likely to arise in the case of glycolysis, cannot be solved using current standard methods. As a result, while we have some intuition of the results for sigmoid benefits (Archetti and Scheuring, 2011, 2012) a full analytical account of the problem of non-linear public goods has been so far beyond the reach of evolutionary game theory (but see Archetti, 2013). To complicate matters further, excessive acidity leads to self-poisoning, and is hence deleterious even for tumor cells (Thomlinson and Gray, 1955; Holash et al., 1999). The benefit function that must be modeled is, therefore, not only non-linear, but also non-monotonic, which complicates the analysis even more.

Here I use a new approach to non-linear public goods games (based on the properties of Bernstein polynomials [used also in the analysis of the dynamics of growth factor production: Archetti, 2013]) to characterize the dynamics and find the equilibria of a multi-player, public goods game with non-linear, non-monotonic benefits that models the scenario of the Warburg effect. This enables us to model the collective benefit arising from glycolysis assuming (unlike previous models) that resistance can evolve in cells with aerobic metabolism, or that resistant cells can forego glycolysis, and that oxygen is not limiting. We will show that, even in these cases, glycolysis can evolve in cancer populations because of the acid-mediated tumor invasion effect, thereby explaining the Warburg effect under general circumstances, and revealing further details of the dynamics of the system that can be useful in planning adaptive therapies.

\section{Model and results}

\subsection{The game}

Cells with glycolytic metabolism can be considered cooperators (C) because they pay a cost $c>0$, due to their inefficient metabolism, leading to slower cell proliferation; the resulting benefit (due to the products of glycolysis that induce environmental acidity; against normal cells) accrues to all cancer cells, including those without glycolysis (defectors, D) that do not pay the cost of an inefficient metabolism. This benefit $b(j)$ is a non-linear function of the number $j$ of $C$ cells in the group; a cell's group is defined by the diffusion range of the products of the glycolysis of the surrounding cells or, more properly, as the range of the benefit resulting from the acidity induced by these cells. Non-linear benefits are generally modeled by a sigmoid function, which allows to describe various types of synergistic effects (Archetti and Scheuring, 2011, 2012; Archetti, 2013); here, however, in order to take into account the possibility that high levels of glycolysis are detrimental to tumor cells (self-poisoning) we must use a double sigmoid function

$b(j)= \begin{cases}b_{1}(j) & j<d \cdot n \\ b_{2}(j) & j \geq d \cdot n\end{cases}$

where

$b_{1}(j)=\left[l_{1}(j)-l_{1}(0)\right] /\left[l_{1}(d \cdot n)-l_{1}(0)\right]$

$b_{2}(j)=1-\left[l_{2}(j, y)-l_{2}(d \cdot n, y)\right] /\left[l_{2}(n, 1)-l_{2}(d \cdot n, 1)\right]$
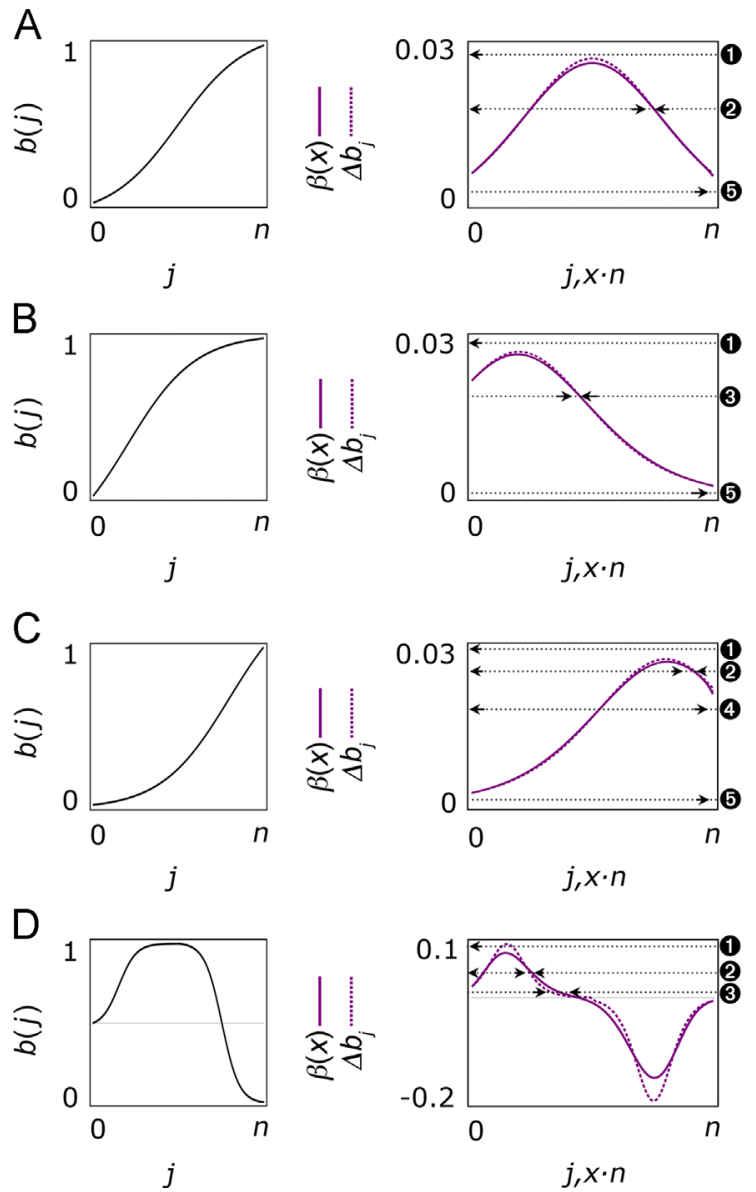

Fig. 1. Possible types of evolutionary dynamics. The benefit of glycolysis $b(j)$ as a function of the number $(j)$ of $C$ cells (left; the continuous line is for ease of visualization) and the corresponding $\beta(x)$ (right: full line) and $\Delta b_{j}$ (right: dashed line). The equilibria are found where $\beta(x)=c$, that is, where the curve intersects the constant line $c$ (dotted arrow; the arrows show the direction of the change); the fraction of glycolytic cells increases if $\beta(x)>c$ and decreases if $\beta(x)<c$. Five types of dynamics are possible, identified by labeled numbered from 1 to 5 : only $x=0$ is stable (type 1 ); both $x=0$ and $x=x_{s}\left(0<x_{s}<1\right)$ are stable (type 2); only $x=x_{s}$ is stable (type 3); both $x=0$ and $x=1$ are stable (type 4 ); only $x=1$ is stable (type 5 ). A-C: no self-poisoning (A: $h=0.2 ; \mathrm{B}: h=0.5 ; \mathrm{C}: h=0.8$; in all cases $d=1, s=5$ ); D: self-poisoning $(d=0.5, s=10, h=0.3, y=2, s 2=10, h 2=0.5)$; in all cases $n=50$.

are the normalized versions of the logistic functions

$l_{1}(j)=\frac{1}{1+e^{\left.s_{1} \cdot\left(h_{1}-(j / n) / d\right)\right)}}$

$l_{2}(j, y)=\frac{y}{1+e^{\left.s_{2} \cdot\left(h_{2}-(j / n-d) /(1-d)\right)\right)}}$

The parameter $d$ describes the value of $j$ at which the benefits of acidity are overcome by its deleterious effects; for $j<d n$, the function is monotonically increasing and has an inflection point at $h_{1}$ and steepness $s_{1}$; for $j>d n$, the function is monotonically decreasing and has an inflection point at $h_{2}$ and steepness $s_{2}$ (with $0<h_{1}, h_{2} \leq 1$ and $s_{1}, s_{2}>0$ ); the additional parameter $y$ measures the maximum damage of self-poisoning. This function is shown graphically in Figs. 1d and 2.

In a large population with no assortment, we can approximate the analysis by assuming an infinite, well-mixed population, and the fitnesses of $C$ and $D$ cells are given by, respectively

$\pi_{C}(x)=\sum_{j=0}^{n-1}\left(\begin{array}{c}n-1 \\ j\end{array}\right) x^{j}(1-x)^{n-1-j} \cdot b(j+1)-c$ 

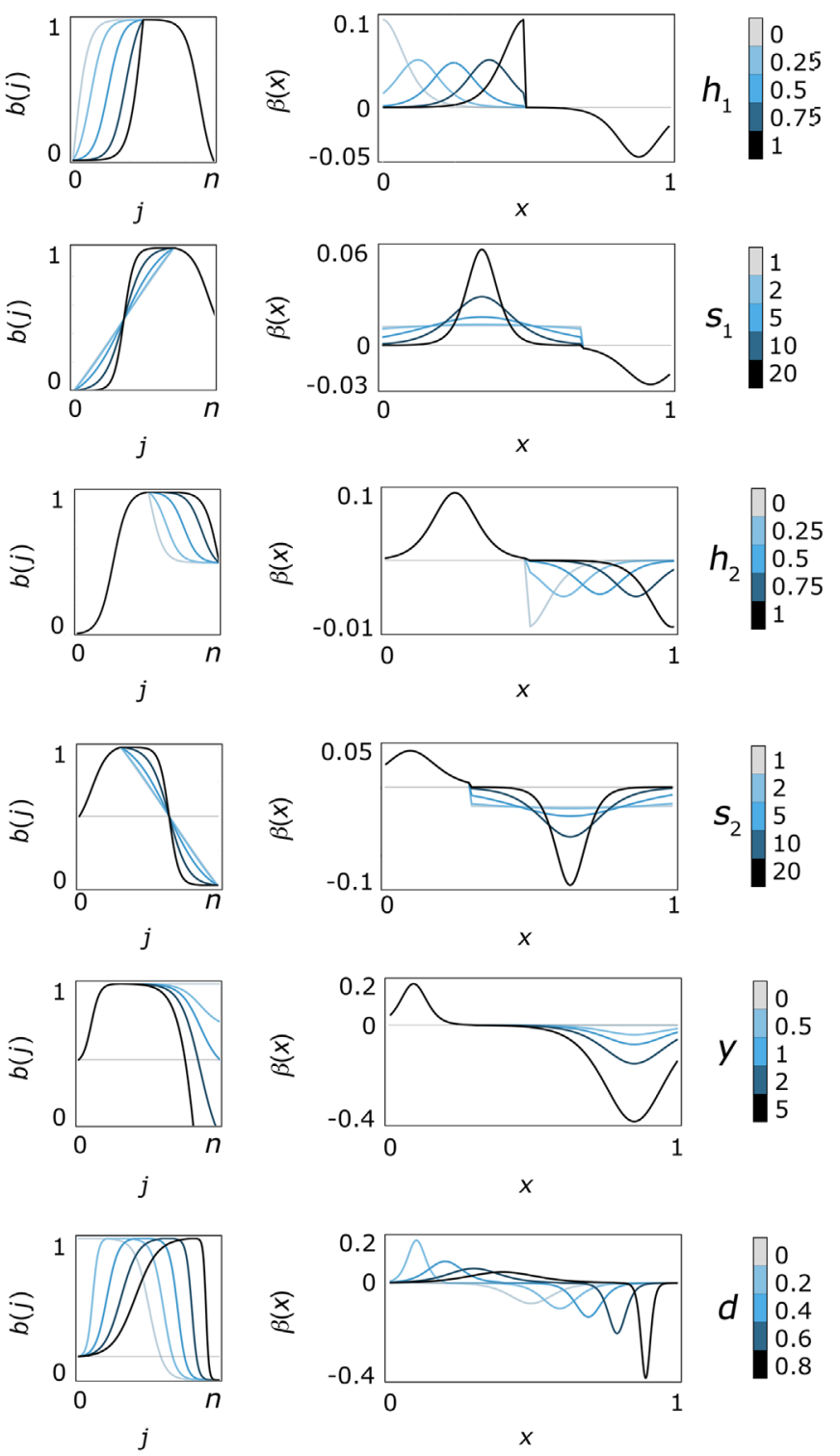

Fig. 2. Effect of the type of benefit of glycolysis on the dynamics. Different types of benefit $b(j)$ as a function of the number $(j)$ of glycolytic cells (left; the continuous line is for ease of visualization) and the corresponding $\beta(x)$ for different values of $h_{1}$ (with $d=0.5, h_{2}=0.8, n=100, s_{1}=10, s_{2}=10, y=1$ ), $s_{1}$ (with $d=0.7, h_{1}=0.5, h_{2}=0.8$, $n=100, s_{2}=5, y=0.5$ ), $h_{2}$ (with $d=0.5, h_{1}=0.5, n=50, s_{2}=10, s=10, y=0.5$ ), $s_{2}$ (with $d=0.3, h_{2}=0.5, h_{1}=0.3, n=100, s_{1}=5, y=2$ ), $y$ (with $d=0.3, h_{2}=0.8, h_{1}=0.3$, $n=50, s_{2}=10, s_{1}=10$ ) and $d$ (with $h_{2}=0.5, h_{1}=0.5, n=50, s_{2}=20, s_{1}=10, y=1.2$ ). Equilibria are obtained, as in Fig. 1, by the intersection of the constant line c (not shown here for clarity) and $\beta(x)$. Note that, because the cost of glycolysis $\mathrm{c}$ is always positive, $y, h_{2}$ and $s_{2}$ (and therefore self-poisoning) have no effect on the position of the equilibria but only on the speed of the dynamics.

$\pi_{D}(x)=\sum_{j=0}^{n-1}\left(\begin{array}{c}n-1 \\ j\end{array}\right) x^{j}(1-x)^{n-1-j} \cdot b(j)$

where $0 \leq x \leq 1$ is the fraction of $C$ cells in the population, Since a $C$ cell pays $a$ cost $c$ that a D cell does not pay, but its group has one more contributor (itself).

\subsection{Dynamics}

In a clonal population, the replicator dynamics (Hofbauer and Sigmund, 1998) of this game is given by

$\dot{x}=x(1-x) \cdot[\beta(x)-c]$ where the fitness difference $\pi_{C}(x)-\pi_{D}(x)$ is written in the form $\beta(x)-c$, and

$\beta(x)=\sum_{j=0}^{n-1}\left(\begin{array}{c}n-1 \\ j\end{array}\right) x^{j}(1-x)^{n-1-j} \cdot \Delta b_{j}$

with $\Delta b_{j}=b((j+1) / n)-b(j / n)$.

This replicator dynamics (5) can be easily shown to have two trivial rest points $x=0$ and $x=1$; further possible interior rest points are found by setting the fitness difference to zero, that is, by the roots of the equation

$\beta(x)-c=0$

Even for the simplest sigmoid function given by the logistic function, an exact analytical solution for (7) is not possible. Because $\beta(x)$ is a polynomial in Bernstein form (Bernstein, 1912; Lorentz, 1953; Phillips, 2003) of the coefficient $\Delta b_{j}$, however, we can resort to the properties of Bernstein polynomials to characterize the dynamics, and to Bernstein theorem to find an approximate solution for the equilibria; a similar approach has been used in the study of growth factor production (Archetti, 2013). In short, because of the variationdiminishing property of Bernstein polynomials we known that the number of internal equilibria of $\beta$ is less than the number of sign changes of $\Delta b$ by an even amount. Moreover, because of the endpoint values property, we know that $\beta(0)=\Delta b_{0}$ and $\beta(1)=\Delta b_{n-1}$. The assumption that the benefit function has the double sigmoid shape defined by (1) implies that there are at most one maximum and one minimum in $(0,1)$. This allows to characterize the dynamics of the system according to the following types (Fig. 2), where $\beta_{\text {MAX }}$ is the maximum value of $\beta(x)$.

- If $c>\beta_{\mathrm{MAX}}$, then $\beta(x)<0 \forall x$, and $x=0$ is the only stable equilibrium and $x=1$ is the only unstable equilibrium.

- If Max $\left[\Delta \mathrm{b}_{0}, \Delta \mathrm{b}_{n-1}\right]<c<\beta_{\operatorname{MAX}}$ then $\beta(x)>0$ for $x_{u}<x<x_{s}$, while $\beta(x)<0$ for $x<x_{u}$ and for $x>x_{s}$; the interior unstable equilibrium $x_{u}$ divides the basins of attraction of the two stable equilibria $x=0$ and $x=x_{s} ; x=1$ is an unstable equilibrium.

- If $\Delta b_{n-1}<c<\Delta b_{0}$ then $\beta(x)>0$ for $x<x_{s}$ and $\beta(x)<0$ for $x>x_{s}$; therefore the unique interior stable equilibrium $x_{s}$ divides the basin of attraction of the two unstable equilibria $x=1$ and $x=0$.

- If $\Delta b_{0}<c<\Delta b_{n-1}$ then $\beta(x)>0$ for $x>x_{u}$ and $\beta(x)<0$ for $x<x_{u}$; therefore the unique interior unstable equilibrium $x_{u}$ divides the basin of attraction of the two stable equilibria $x=1$ and $x=0$.

- If $c<\operatorname{Min}\left[\Delta b_{0}, \Delta b_{n-1}\right]$ then $\beta(x)>0 \forall x$, and $x=1$ is the only stable equilibrium.

The analysis above holds for any double sigmoid functions (not limited to the double logistic function). The latter two types are not possible with self-poisoning (Fig. 1d).

\subsection{Equilibria}

We can find the equilibria numerically by (7) (Fig. 3). Since by Bernstein theorem (Bernstein, 1912) $\beta(x)$ converges uniformly to $\Delta b_{j}$ (the forward difference of the benefit function with spacing $1 / n$ ) in $[0,1]$, we can also approximate the solution by setting $\Delta b_{j}$ to zero. If we exclude self-poisoning $(d=1)$ we can find an approximate analytical solution (see Archetti, 2013) by $b^{\prime}(x)=\mathrm{cn}$, since $\Delta b_{j} \approx(1 /$ $n) b^{\prime}(j / n)$ and since for any $x, j / n$ converges in probability to $x$; in this case the internal equilibria are given therefore by (for the logistic function)

$x_{ \pm}=h_{1}-\frac{s_{1}}{n} \log \left[\frac{1 \pm \sqrt{1-4 c s_{1} \cdot B}}{2 c s_{1} \cdot B}-1\right]$ 


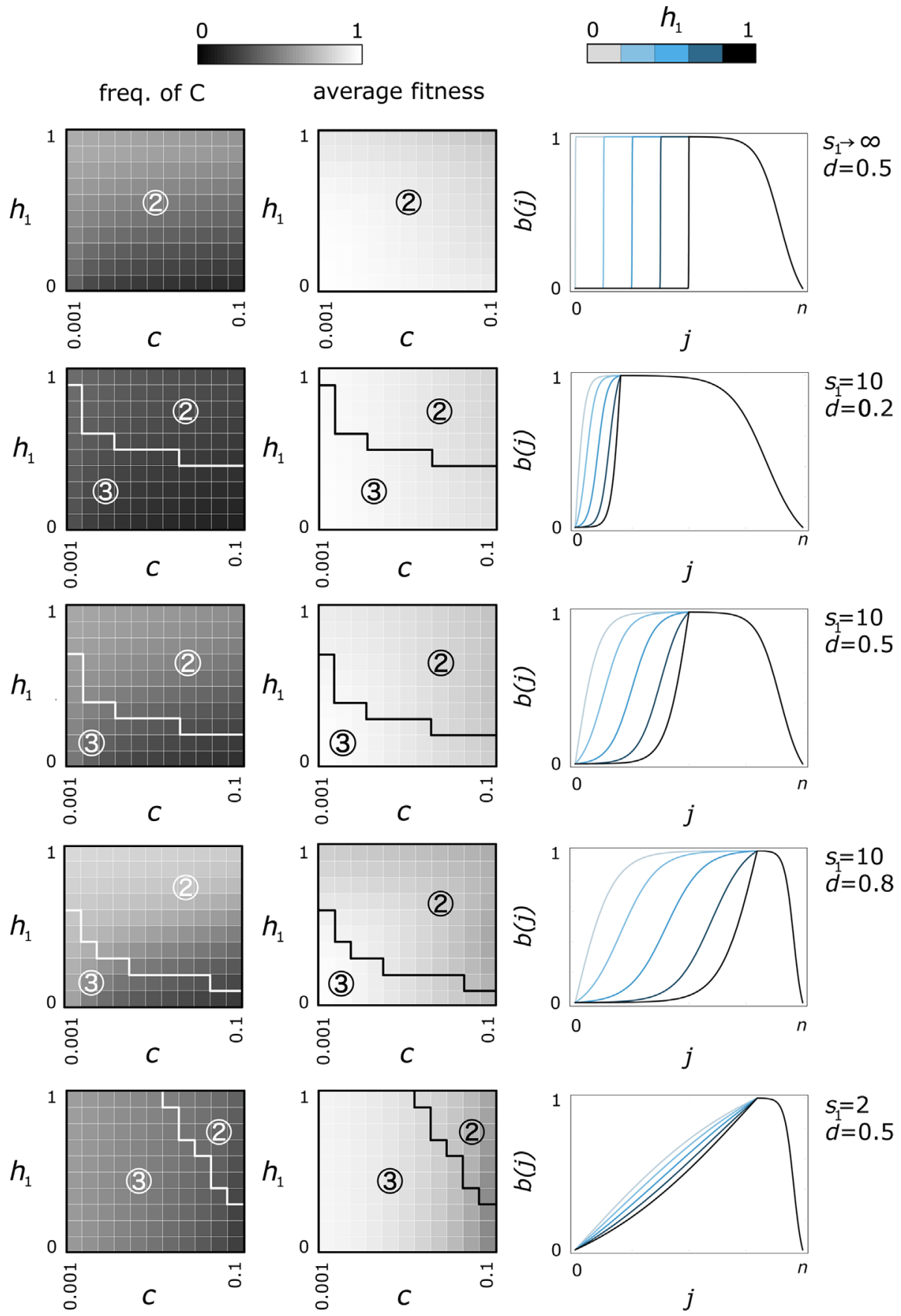

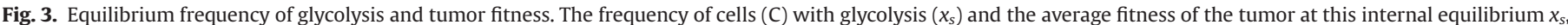

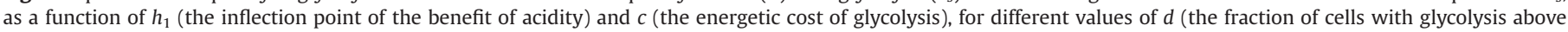

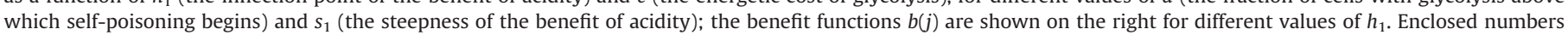
show parameter spaces corresponding to different types of dynamics (type 2 and 3 - see Fig. 1 ). Other parameters used: $n=20, s_{2}=10, h_{2}=0.8, y=1$.

with $x_{s}=x_{-}$for the stable equilibrium and $x_{u}=x_{+}$for the unstable equilibrium, where $B=l_{1}(1)-l_{1}(0)$. As predicted by the analysis of the dynamics, the glycolytic type can go to extinction under certain circumstances, but it can also be maintained at equilibrium in coexistence with the non-glycolytic type (Fig. 3). If self-poisoning occurs, as noted above, the glycolytic type will not go to fixation.

\subsection{Effect of the parameters}

The parameters that describe self-poisoning $\left(s_{2}, h_{2}, y\right)$ affect only the speed of change when $x>x_{s}$, but not the equilibria
(Fig. 2). The type of dynamics depends on how steep the benefit function is at $h_{1}$ (that is, on $s_{1}$ ), and on the fraction of $C$ cells necessary before self-poisoning occurs $(d)$; bistability occurs more often when $d$ and $s_{1}$ are high (Fig. 3). In all cases, increasing the cost $c$ reduces the frequency of $C$ types and average fitness at equilibrium (Fig. 3), which is intuitive. The effect of $h_{1}$ are less straightforward: reducing $h_{1}$ leads to a lower value of the frequency of $\mathrm{C}$ at equilibrium, but this does not necessarily translate to a lower fitness for the population (Fig. 3), since a lower amount of $C$ types is necessary to achieve a benefit; furthermore, very low (or very high) $h_{1}$ values allow an internal 
equilibrium to exist even for costs $(c)$ that lead to no internal equilibrium at intermediate values of $h_{1}$ (Fig. 2).

\subsection{Effect of changes in acidity}

How does tumor fitness change in response to externally imposed (not produced by the cancer cells themselves) changes in acidity? Consider a change in acidity equal to that that would be produced by a further fraction $\zeta$ of C cells (Fig. 4$)$ : a negative change $(\zeta<0)$ clearly reduces the benefit for the cancer cell population because of lower acidity; a positive change $(\zeta>0)$ can reduce tumor fitness if the change is large enough that it leads to self-poisoning; if the change is small, however, it can lead to an increase in fitness (especially for low values of $h_{1}$ and high values of $c$; see Fig. 4a). This is because the equilibrium frequency of $C$ cells is generally inefficient, that is, it leads to a suboptimal public good being produced (the maximum benefit is achieved for slightly higher frequencies of $C$ ): increasing the fraction of $C$ cells ( $x$, which in this case is mimicked by the additional exogenous acidity $\zeta$ ) can increase average population fitness, as long as the increase is not too large (Fig. 4b).

\section{Discussion}

We have analyzed a scenario in which glycolysis is costly (because energetically inefficient, thus leading to slower proliferation) but produces a collective benefit (acidity) for all cancer cells (glycolytic or not). Glycolysis is, therefore, a form of cooperation among cancer cells for increased acidity, which confers an advantage to the tumor as a group. The resulting dynamics enables glycolysis to persist even under non-limiting oxygen conditions (that is, even if glycolysis is inefficient) under many circumstances because the benefit of acidification is a non-linear function of the frequency of glycolytic cells. A typical outcome of this dynamics (Fig. 1, case 2) is that too few glycolytic cells would lead to not enough acidity for glycolysis to be useful, and its intrinsic energetic cost would make it go extinct from the population; the marginal additional benefit of further acidity in the presence of too many other glycolytic cells, on the other hand, would not be worth the cost in terms of inefficient metabolism (note that this would be true even in the absence of selfpoisoning); glycolysis, however, confers a higher fitness to an individual cell if there are neither too many nor too few other glycolytic cells; the result is that, unless the cost of glycolysis is too high, glycolytic cells can persist at intermediate frequencies in the tumor even when glycolysis is inefficient, that is, even under normal oxygen concentrations.

This leaves unexplained the problem of the origin of glycolysis, since a critical fraction of glycolytic cells is necessary, in this case, to lead a population to the basin of attraction of this stable equilibrium. In other words, the first glycolytic cell will pay a cost without gaining any benefit, and will therefore not invade. In other cases, however, this origin problem does not exist, either because acidification confers diminishing returns without self-poisoning (Fig. 1, case 3) or because the cost of glycolysis is low (Fig. 1, case 5);

A

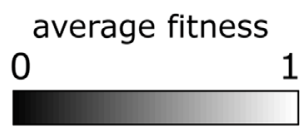

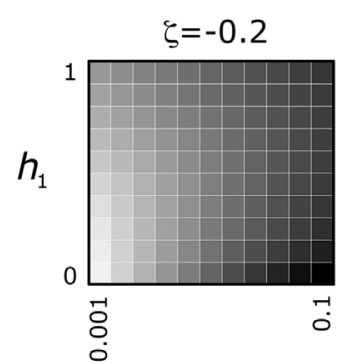

$C$

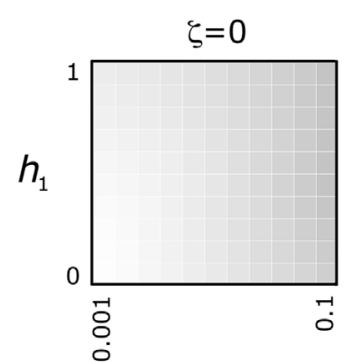

C

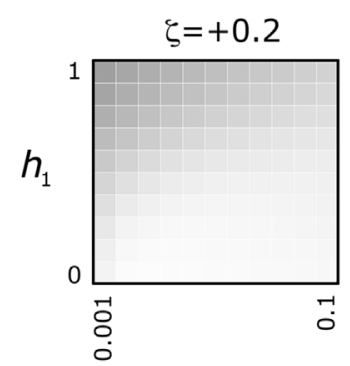

C

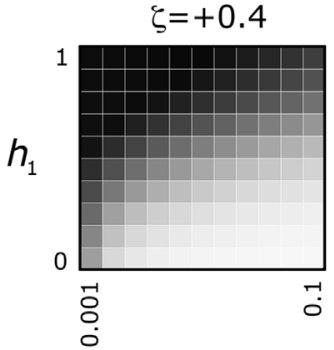

C

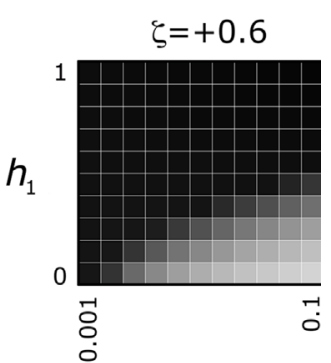

C

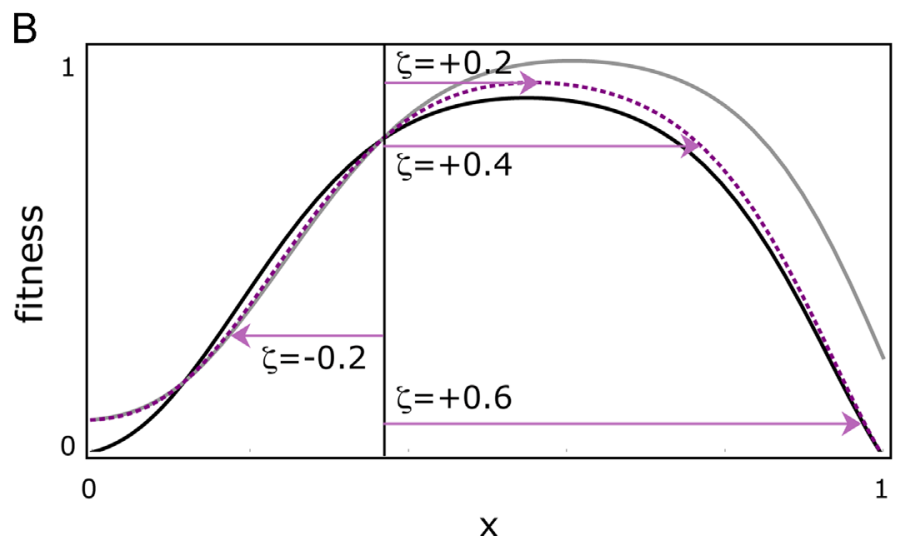

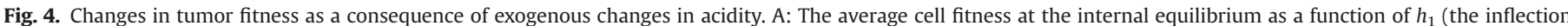

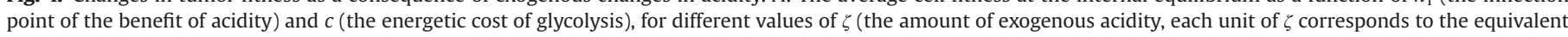

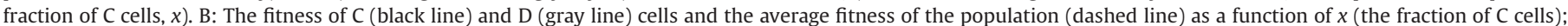
arrows show the effects of changes in $\zeta ; c=0.1, h_{1}=0.5$. In all cases: $n=20, s_{1}=10, s_{2}=10, h_{2}=0.8, y=1, d=0.5$. 
in this case the population will move towards a higher frequency of glycolytic cells, and the glycolytic type can even go to fixation; if oxygen concentrations then revert to normal, the cost of glycolysis will increase, leading the system to a stable coexistence of glycolytic and non-glycolytic types (Fig. 1, from case 5 to case 2). The problem of the origin can also be solved by assuming that cells increase their anaerobic metabolism, through increase in glycolysis, during episodes of hypoxia or when far from vascularized tissue, for example during tumor expansion not accompanied by angiogenesis (Smallbone et al., 2007).

Studying the dynamics of glycolysis in terms of collective interactions between cancer cells for the acidification of the extra-cellular environment solves a problem that previous models (Gatenby and Gawlinski, 1996; Patel et al., 2001; Smallbone et al., 2005, 2007; Basanta et al., 2008, 2011) of the acid-mediated tumor invasion hypothesis (Gatenby and Gawlinski, 1996, 2003; Gatenby and Gillies, 2004, 2007, Gillies and Gatenby 2007) had left unsolved: why does the Warburg effect occur under normal oxygen concentrations? Considering the products of glycolysis as a diffusible public good with nonlinear (non-monotonic) benefits, therefore, enables us to fully explain the Warburg effect.

Glycolysis and the acid-mediated tumor invasion hypothesis have implications for treatment, and it has been suggested that manipulating acidity can lead to adaptive anti-cancer therapies (Gatenby and Gillies, 2007; Robey et al., 2009; Ibrahim Hashim et al., 2011, 2012; Martin et al., 2011, 2012). Reducing acidity will lead to an immediate reduction in fitness for the tumor because of a reduction in the benefits of acidity; this is intuitive (Fig. 4). Less intuitively, analyzing the Warburg effect as a public goods game reveals also that increasing acidity can have opposite effects: while a large increase can lead to self-poisoning, and therefore be a potential anti-cancer strategy (as suggested by Smallbone et al., 2010), a mild increase may lead to a higher fitness for the tumor (Fig. 4). In the short term, therefore, modifying acidity may lead to a reduction of the proliferation rate of the tumor. The long term effect, on the other hand, depend on how large, fast and persistent the change is; the population may simply readapt to the new acidity conditions: the frequency of $\mathrm{C}$ types will change, leading the population to a new equilibrium (that will have improved proliferation), unless the change (the external increase in acidity) is such that, when the original acidity is restored, the equilibrium fraction of $C$ is so low that the $C$ type goes extinct.

We must keep in mind that certain features of the Warburg effect have been ignored in the model. Notably, we have assumed that cells can be of two different, fixed types (glycolytic or not). In reality, a cell could modulate the amount of glycolysis based on external conditions. That is, we have modeled a purely evolutionary dynamics in which changes occur only because of clonal selection. Cells can, however, also have more immediate physiological responses to environmental conditions that resemble the "best response" dynamics of rational players used in economic game theory, rather than the adaptive dynamics used in evolutionary game theory (adopted here). While the type of equilibria are unlikely to change, the dynamics would be slightly different from the one described here.

Mutations that confer resistance to therapies designed to inhibit glucose metabolism can clearly evolve (Gatenby and Gillies, 2007). Resistance to anti-cancer therapies arises because cancer progression is an evolutionary process of clonal selection. Studying glycolysis as a multiplayer public goods game for the production of a non-linear (non-monotonic) diffusible good can not only explain the adaptive value of the Warburg effect, but also reveal properties of the evolutionary dynamics that can be used to devise or optimize adaptive therapies.

\section{References}

Archetti, M., 2009a. The volunteer's dilemma and the optimal size of a social group. Journal of Theoretical Biology 261, 475-480.

Archetti, M., 2009b. Cooperation as a volunteer's dilemma and the strategy of conflict in public goods games. Journal of Evolutionary Biology 22, 2192-2200.

Archetti, M., 2013. Evolutionary game theory of growth factor production: implications for tumor heterogeneity and resistance to therapies. British Journal of Cancer 109, 1056-1062.

Archetti, M., Scheuring, I., 2011. Coexistence of cooperation and defection in public goods games. Evolution 65, 1140-1148.

Archetti, M., Scheuring, I., 2012. Review: Evolution of cooperation in one-shot social dilemmas without assortment. Journal of Theoretical Biology 299, 9-20.

Basanta, D., Scott, J.G., Rockne, R., et al., 2011. The role of IDH1 mutated tumour cells in secondary glioblastomas: an evolutionary game theoretical view. Physical Biology 8, 015016.

Basanta, D., Simon, M., Hatzikirou, H., Deutsch, A., 2008. Evolutionary game theory elucidates the role of glycolysis in glioma progression and invasion. Cell Proliferation 41, 980-987.

Beckner, M.E., et al., 2005. Glycolytic glioma cells with active glycogen synthase are sensitive to PTEN and inhibitors of P13K and gluconeogenesis. Laboratory Investigation 85, 1457-1470.

Bergers, G., Hanahan, D., 2008. Modes of resistance to anti-angiogenic therapy. Nature Reviews Cancer 8, 592-603.

Bernstein, S., 1912. Démonstration du théorème de Weierstrass fondée sur le calcul des probabilities. Communications of the Kharkov Mathematical Society 13, 1-2.

Bhujwalla, Z.M., et al., 2002. Combined vascular and extracellular pH imaging of solid tumors. NMR in Biomedicine 15, 114-119.

Buchler, P., et al., 2003. Hypoxia-inducible factor 1 regulates vascular endothelial growth factor expression in human pancreatic cancer. Pancreas 26, 56-64.

Cairns, J., 1975. Mutation selection and the natural history of cancer. Nature 255, 197-200.

Casciari, J.J., Sotirchos, S.V., Sutherland, R.M., 1992. Variations in tumor growth rates and metabolism with oxygen concentration, glucose concentration, and extracellular pH. Journal of Cellular Physiology 151, 386-394.

Crespi, B., Summers, K., 2005. Evolutionary biology of cancer. Trends in Ecology \& Evolution 20, 545-552.

Dairkee, S.H., Deng, S.H., Stampfer, M.R., Waldman, R.M., Smith, H.S., 1995. Selective cell culture of primary breast cancer. Cancer Research 55, 2516-2519.

Dewhirst, M.W., Secomb, T.W., Ong, E.T., Hsu, R., Gross, J.F., 1994. Determination of local oxygen consumption rates in tumors. Cancer Research 54, 3333-3336.

Gambhir, S.S., 2002. Molecular imaging of cancer with positron emission tomorgraphy. Nature Reviews Cancer 2, 891-899.

Gatenby, R., Gillies, R.J., 2004. Why do cancers have high aerobic glycolysis? Nature Reviews Cancer 4, 891-899.

Gatenby, R., Gillies, R.J., 2007. Glycolysis in cancer: a potential target for therapy. International Journal of Biochemistry \& Cell Biology 39, 1358-1366.

Gatenby, R.A., Gawlinski, E.T., 1996. A reaction-diffusion model of cancer invasion. Cancer Research 56, 5745-5753.

Gatenby, R.A., Gawlinski, E.T., 2003. The glycolytic phenotype in carcinogenesis and tumor invasion: insights through mathematical models. Cancer Research 63, 3847-3854.

Greaves, M., Maley, C.C., 2012. Clonal evolution in cancer. Nature 481, 306-313.

Griffiths, J.R., McIntyre, D.J., Howe, F.A., Stubbs, M., 2001. Why are cancers acidic? A carrier-mediated diffusion model for $\mathrm{H}^{+}$transport in the interstitial fluid. Novartis Foundation Symposium 240, 46-62.

Griguer, C.E., et al., 2005. Glucose metabolism heterogeneity in human and mouse malignant glioma cell lines. Journal of Neuro-Oncology 74, 123-133.

Hauert, C., Michod, F., Nowak, M.A., Doebeli, M., 2006. Synergy and discounting of cooperation in social dilemmas. Journal of Theoretical Biology 239, 195-202.

He, X., et al., 2004. Hypoxia increases heparanase-dependent tumor cell invasion, which can be inhibited by antiheparanase antibodies. Cancer Research 64 3928-3933.

Helmlinger, G., Yuan, F., Dellian, M., Jain, R.K., 1997. Interstitial pH and pO gradients in solid tumors in vivo: high-resolution 2 measurements reveal a lack of correlation. Nature Medicine 3, 177-182.

Hofbauer, J., Sigmund, K., 1998. Evolutionary Games and Population Dynamics. Cambridge University Press, Cambridge, UK.

Holash, J., Maisonpierre, P.C., Compton, D., Boland, P., Alexander, C.R., Zagzag, D., Yancopoulos, G.D., Wiegand, S.J., 1999. Vessel co-option, regression, and growth in tumors mediated by angiopoietins and VEGF. Science 284, 1994-1998.

Ibrahim Hashim, A., Cornnell, H.H., Abrahams, D., Lloyd, M., Bui, M., Gillies, R.J., Gatenby, R.A., 2012. Systemic buffers inhibit carcinogenesis in TRAMP mice. Journal of Urology 188, 624-631.

Ibrahim Hashim, A., Cornnell, H.H., Coelho Ribeiro Mde, L., Abrahams, D., Cunningham, J., Lloyd, M., Martinez, G.V., Gatenby, R.A., Gillies, R.J., 2011. Reduction of metastasis using a non-volatile buffer. Clinical and Experimental Metastasis 28 $841-849$.

Kelloff, G.J., et al., 2005. Progress and promise of FDG-PET imaging for cancer patient management and oncologic drug development. Clinical Cancer Research 11, 2785-2808.

Krogh, A., 1919. The number and distribution of capillaries in muscles with calculations of the oxygen pressure head necessary for supplying the tissue. Journal of Physiology 52, 409-415. 
Krtolica, A., Ludlow, J.W., 1996. Hypoxia arrests ovarian carcinoma cell cycle progression, but invasion is unaffected. Cancer Research 56, 1168-1173.

Kunkel, M., et al., 2003. Overexpression of Glut-1 and increased glucose metabolism in tumors are associated with a poor prognosis in patients with oral squamous cell carcinoma. Cancer 97, 1015-1024.

Lardner, A., 2001. The effects of extracellular pH on immune function. Journal of Leukocyte Biology 69, 522-530.

Lorentz, G.G., 1953. Bernstein Polynomials. University of Toronto Press, Toronto, Canada.

Martin, N.K., Gaffney, E.A., Gatenby, R.A., Gillies, R.J., Robey, I.F., Maini, P.K., 2011. A mathematical model of tumour and blood pHe regulation: The HCO3-/CO2 buffering system. Mathematical Biosciences 230, 1-11.

Martin, N.K., Robey, I.F., Gaffney, E.A., Gillies, R.J., Gatenby, R.A., Maini, P.K., 2012. Predicting the safety and efficacy of buffer therapy to raise tumour pHe: an integrative modelling study. British Journal of Cancer 106, 1280-1287.

Merlo, L.M.F., Pepper, J.W., Reid, B.J., Maley, C.C., 2006. Cancer as an evolutionary and ecological process. Nature Reviews Cancer 6, 924-935.

Mochiki, E., et al., 2004. Evaluation of 18F-2-deoxy-2-fluoro-D-glucose positron emission tomography for gastric cancer. World Journal of Surgery 28, 247-253.

Motro, U., 1991. Co-operation and defection: playing the field and ESS. Journal of Theoretical Biology 151, 145-154.

Nowell, P.C., 1976. The clonal evolution of tumor cell populations. Science 194, 23-28.

Park, H.J., Lyons, J.C., Ohtsubo, T., Song, C.W., 1999. Acidic environment causes apoptosis by increasing caspase activity. British Journal of Cancer 80, 1892-1897.

Patel, A.A., Gawlinski, E.T. Lemieux, S.K., Gatenby, R.A., 2001. A cellular automaton model of early tumor growth and invasion: the effects of native tissue vascularity and increased anaerobic tumor metabolism. Journal of Theoretical Biology 213, 315-331.

Pauwels, E.K., Sturm, E.J., Bombardieri, E., Cleton, F.J., Stokkel, M.P., 2000. Positronemission tomography with [18F]fluorodeoxyglucose. Part I. Biochemical uptake mechanism and its implication for clinical studies. Journal of Cancer Research and Clinical Oncology 126, 549-559.

Phillips, G.M., 2003. Interpolation and Approximation by Polynomials. Springer, New York, USA (Chapter 7).

Postovit, L.M., Adams, M.A., Lash, G.E., Heaton, J.P., Graham, C.H., 2004. Nitric oxidemediated regulation of hypoxia- induced B16F10 melanoma metastasis. International Journal of Cancer 108, 47-53.

Postovit, L.M., Adams, M.A., Lash, G.E., Heaton, J.P., Graham, C.H., 2002. Oxygenmediated regulation of tumor cell invasiveness. Involvement of a nitric oxide signaling pathway. Journal of Biological Chemistry 277, 35730-35737.
Rajendran, J.G., et al., 2003. [(18)F]FMISO and [(18)F]FDG PET imaging in soft tissue sarcomas: correlation of hypoxia, metabolism and VEGF expression. European Journal of Nuclear Medicine and Molecular Imaging 30, 695-704.

Robey, I.F., Baggett, B.K., Kirkpatrick, N.D., Roe, D.J., Dosescu, J., Sloane, B.F., Hashim, A.I., Morse, D.L., Raghunand, N., Gatenby, R.A., Gillies, R.J., 2009. Bicarbonate increases tumor $\mathrm{pH}$ and inhibits spontaneous metastases. Cancer Research 69, 2260-2268.

Rohzin, J., Sameni, M., Ziegler, G., Sloane, B.F., 1994. Pericellular pH affects distribution and secretion of cathepsin B in malignant cells. Cancer Research 54, 6517-6525.

Rubin, H.J., 1971. pH and population density in the regulation of animal cell multiplication. Journal of Cell Biology 51, 686-702.

Schornack, P.A., Gillies, R.J., 2003. Contributions of cell metabolism and $\mathrm{H}^{+}$ diffusion to the acidic $\mathrm{pH}$ of tumors. Neoplasia 5, 135-145.

Shi, Q., Le, X., Wang, B., Abbruzzese, J.L., Xiong, Q., He, Y., Xie, K., 2001. Regulation of vascular endothelial growth factor expression by acidosis in human cancer cells. Oncogene 20, 3751-3756.

Silva, A.S., Gatenby, R.A., Gillies, R.J., Yunes, J.A., 2010. A quantitative theoretical model for the development of malignancy in ductal carcinoma in situ. Journal of Theoretical Biology 262, 601-613.

Smallbone, K., Gatenby, R.A., Gillies, R.J., Maini, P.K., Gavaghan, D.J., 2007. Metabolic changes during carcinogenesis: potential impact on invasiveness. Journal of Theoretical Biology 244, 703-713.

Smallbone, K., Gavaghan, D.J., Gatenby, R.A., Maini, P.K., 2005. The role of acidity in solid tumour growth and invasion. Journal of Theoretical Biology 235, 476-484.

Smallbone, K., Maini, P.K., Gatenby, R.A., 2010. Episodic, transient systemic acidosis delays evolution of the malignant phenotype: Possible mechanism for cance prevention by increased physical activity. Biology Direct 5, 22.

Thomlinson, R.H., Gray, L.H., 1955. The histological structure of some human lung cancers and the possible implications for radiotherapy. British Journal of Cancer 9, 539-549.

Warburg, O., 1930. Ueber den Stoffwechsel der Tumoren. Constable, London.

Williams, A.C., Collard, T.J., Paraskeva, C., 1999. An acidic environment leads to p53 dependent induction of apoptosis in human adenoma and carcinoma cell lines: implications for clonal selection during colorectal carcinogenesis. Oncogene 18 3199-3204.

Wojtkowiak, J.W., Rothberg, J.M., Kumar, V., Schramm, K.J., Haller, E., Proemsey, J.B. Lloyd, M.C., Sloane, B.F., Gillies, R.J., 2012. Chronic autophagy is a cellular adaptation to tumor acidic pH microenvironments. Cancer Research 72 3938-3947. 